\title{
NEARLY MICROARCSECOND PRECISION DIFFERENTIAL ASTROMETRY +
}

J. Marcaide1, I. Shapiro2, J. Ba112, N. Barte12, T. Clark3, B. Corey 4 , M. Gorenstein ${ }^{2}$, R. Preston 5 , M. Ratner ${ }^{2}$, A. Rogers ${ }^{4}$, and A. Whitney 4

${ }^{1}$ Max-Planck-Institut für Radioastronomie, Bonn, F.R.G.

${ }^{2}$ Harvard-Smithsonian Center for Astrophysics, Cambridge, Mass.

${ }^{3}$ Goddard Space Flight Center, Greenbelt, Maryland

${ }^{4}$ Haystack Observatory, Westford, Mass.

${ }^{5}$ Jet Propulsion Laboratory, Pasadena, Calif.

In studies of extragalactic radio sources with multiple compact components the determination of which components, if any, are stationary and which moving is of importance. In order to learn about the radio properties of the individual components it is also relevant to be able to register maps made at several wavelengths. Both tasks are usually not possible with VLBI because of the irrecoverable corruption of the fringe phase introduced by the propagation medium and the instrumentation. How ever, when two or more compact radio sources are separated by only a small angle from each other difference techniques can be used to help tackle both questions.

In this contribution we present relative position determinations of the radio sources $1038+528$ A, B (see Marcaide et al. in these Proceedings) at several wavelengths for a series of observations from November 1979 through March 1981. The separation of the sources was measured between reference points chosen in each quasar map defined by the positions of the strongest CLEAN components. The observable used was the difference phase-delay corrected for the source structure and the fringe ambiguity. The details of the technique have been discussed by Marcaide (1982) and Marcaide and Shapiro (1983).

The results of our experiments are shown in Fig. 1. The most precise relative position determination was obtained from data from March 1981 at $\lambda 3.6 \mathrm{~cm}$ : The standard deviation was under 4 microarcseconds, which corresponds to a phase-delay rms postfit residual under 2 picoseconds, or equivalently to about half a millimeter of light travel path. A comparison of this separation with that obtained from data from November 1979 at the same wavelength shows no indication of relative motion of the reference points in the maps with an upper 1 imit of 18 microarcsecond/year. Such a limit is consistent with the cosmological interpretation of the

+ Discussion on page 467 361

R. Fanti et al. (eds.), VLBI and Compact Radio Sources, 361-362.

(i) 1984 by the IAU. 
redshifts. However, such a small separation rate computed from only two epochs has to be viewed with caution (see Marcaide and Shapiro (1983) for a related discussion).

Using this broader data base we confirm the result presented by Marcaide et al. elsewhere in these Proceedings that the separation between the reference points in the maps of the $A$ and $B$ quasars is less at longer wavelengths of observation than at short wavelengths. We think this result is due to a wavelength-dependent location of the peak of brightness of the A quasar.

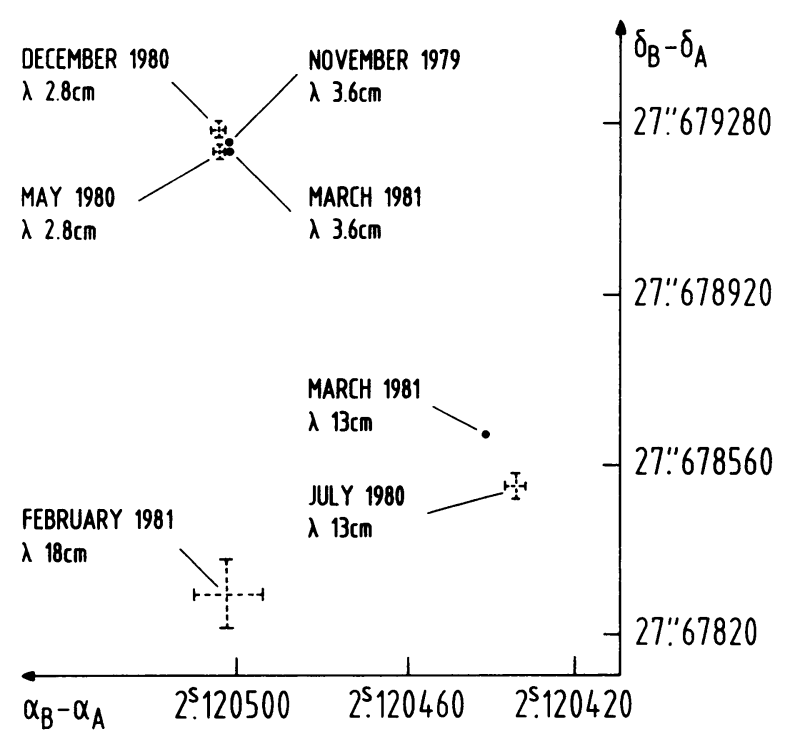

Figure 1:

Positions of the reference points chosen in the map of $1038+528 \mathrm{~B}$ relative to the reference points chosen in A (at the origin). The dashed error indicates that some assumption on the quasar structures was needed due to lack of information from the experiment itself. The displacement of the solution for $\lambda 18 \mathrm{~cm}$ observations with respect to the solutions for $\lambda 13 \mathrm{~cm}$ is likely due to use of an extrapolation of the structure at $\lambda 13 \mathrm{~cm}$ to that at $\lambda 18 \mathrm{~cm}$ based on an incorrect spectral index estimate for one source feature.

\section{REFERENCES}

Marcaide, J.M.: 1982, Ph.D. thesis, Massachusetts Institute of Technology Marcaide, J.M. and Shapiro, I.I.: 1983, Astron. J. (to appear in August 1983 issue) 